Working

Paper

Department

of Economics

Ca' Foscari University of

Venice

Paola Lanaro

Giovanni Favero

Teaching urban history in Italian Universities 


\title{
Teaching urban history in Italian Universities
}

\section{Paola Lanaro and Giovanni Favero}

University of Venice

\begin{abstract}
This paper presents the situation of Urban History teaching in Italian universities, using the results of a web search and of an inquiry performed among Italian teachers by means of a form distribution and collection.

The paper has been presented at the European Association of Urban History conference, Athens, October 27-29, 2004, in the session on Teaching urban history from medieval to modern, coordinated by Richard Rodger and Denis Menjot. It is under press in a collective volume published by the Center for Urban History, Leeds.
\end{abstract}

\section{Keywords}

urban history, teaching, Italy

JEL Codes

I230, I210, N010

Address for correspondence:

Giovanni Favero

Department of Economics Ca’ Foscari University of Venice

Cannaregio 873, Fondamenta S.Giobbe

30121 Venezia - Italy

Phone: (++39) 0412349165

Fax: (++39) 0412349176

e-mail: gfavero@unive.it

This Working Paper (o QD se in inglese) is published under the auspices of the Department of Economics of the Ca' Foscari University of Venice. Opinions expressed herein are those of the authors and not those of the Department. The Working Paper series (o QD se in inglese) is designed to divulge preliminary or incomplete work, circulated to favour discussion and comments. Citation of this paper should consider its provisional character.

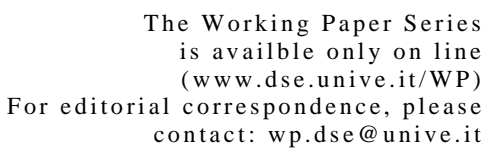

is availble only on line

w.dse.unive.it/WP)

contact:wp.dse@unive.it
Department of Economics

Ca' Foscari University of Venice

Cannaregio 873, Fondamenta San Giobbe

30121 Venice Italy

Fax: ++390412349210 


\section{Teaching urban history in Italian Universities}

This contribution displays the results of a countrywide inquiry on Urban History courses (in all its eventual specific denominations) activated in Italian Universities.

We circulated among the Italian Urban History Association (AISU) members and (with lesser results) among teachers in different disciplines the enclosed form containing questions on teachers' working and academic position, on the course level, number of students, duration and contents, with particular attention to the chronological scope and to textbooks in use. The principal aim of the inquiry was to draw a first map of Urban History teaching in Italy and to point out peculiarities or problems. We collected 22 relevant answers from a universe of about 70 activated courses, as resulting from an exhaustive web search. Nevertheless, we can consider the sample of answers representative for some important variables, except the distribution of courses among different Universities and Faculties.

As far as this latter is concerned, the internet overview allows to appreciate the leading role of some important research poles (Rome and Turin with 8 courses, Naples and Venice with 6, Milan and Catania with 5, Bologna with 4), mostly seat of Polytechnics (Turin and Milan) or Faculties of Architecture and Schools of Archeology (Catania and Bologna), in fostering the activation of Urban History courses. Those are mainly given inside the Faculties of Architecture (58\%); the remaining are mostly inside the Faculties of Letters or Humanities in general (37\%), and only a few in the Faculties of Economics (3\%), Law (1\%) and Engineering (1\%), 
as the table below shows. Denominations spread from History of the City or Urban History (prevailing with 54\% of the courses, comprising different periods and often with explicit attention for the territory, or seldom for archeological, social, economic topics) to History of Urban Studies (35\%, mainly in Faculties of Architecture, sometimes with theoretical or other special features), with less frequent geographic courses with reference to landscape and settlement in their denomination (11\%, mainly in Faculties of Letters).

As we said, the sample of collected answers completely misses the geographical distribution of courses, overrepresenting some local realities. The bias is due to the features of the AISU network, mobilized for the distribution of the questionnaire: it involved only part of the teachers keeping Urban History courses, and among them teachers attaching more importance to this field were autoselected. Anyway, the sample reflects well the distribution of courses among Faculties, although it overrepresents some minor ones, such as Economics and Law. The opposite thing happens with denominations: History of the City and of Urban Studies are well represented, while Landscape Studies is underrepresented.

From the point of view of teachers' academic position, the collected answers show the prevailing presence of Full Professors and Assistant Professors in teaching Urban History (35\% each), with fewer Associate Professors and temporary teachers (15\% each). Courses have an average duration of 50 hours (10 credits), and are mainly activated at the first level degree (65\%, mostly kept by Assistant Professors) and at the second level degree (25\%, almost all kept by Full Professors), and only in few cases inside MA (Pisa) and $\mathrm{PhD}$ programs (Rome, Turin and Venice). The average number of attending students is 45 . It is likely that the high number of students and high duration of courses is correlated with the autoselection of teachers attaching importance to Urban History courses.

The chronological scope of contents is widely differentiated: Modern and Early Modern periods are covered in more than half of the courses, but this percentage falls under 50\% for Middle Ages and under 30\% for Ancient Times. In the great variety of chosen textbooks, some titles are frequently met; this could be a sign of the publication of specific handbooks for Urban History.

We collected also further answers about Urban History topics treated inside different teachings; even if not representative, those answers show that History of Architecture for architects or engineers, and general History in other Faculties, such 
as Letters, Economics, Sociology, often cover Urban History subjects. 
Associazione Italiana di Storia Urbana

Questionario sulla didattica della Storia Urbana nelle Università italiane

Docente

\begin{tabular}{lll}
\hline Nome & COGNOME & Qualifica
\end{tabular}

Sede

\begin{tabular}{ll}
\hline Ateneo & Dipartimento
\end{tabular}

Denominazione del corso

Eventuali corsi monografici di Storia della città all'interno di corsi generali con altra denominazione:

Livello

Facoltà

Corso di laurea

Durata

\begin{tabular}{ll}
\hline Crediti Ore &
\end{tabular}

Ambito cronologico:

Antichità / Medioevo / Età Moderna / Età Contemporanea / Tutto

Programma del corso:

Testi adottati:

Numero di studenti frequentanti

Titoli di eventuali tesi di laurea o di dottorato in Storia Urbana in corso o discusse:

Accordi di scambio di studenti e/o docenti attivati nell'ambito dei Programmi Erasmus/Socrates o altro che comprendono corsi di Storia urbana:

Eventuale interesse a partecipare a iniziative e convegni dell'AISU: 
Italian Association of Urban History

Questionnaire on teaching Urban History in Italian Universities

Teacher

\begin{tabular}{lll}
\hline First name & SURNAME & Academic position
\end{tabular}

Seat

University Department

Course denomination

Eventual special Urban History topics inside general courses with different denomination:

Level

First level / Second level / Master degree / PhD

Faculty

Curriculum

Duration

\begin{tabular}{ll}
\hline Credits & Hours
\end{tabular}

Chronological scope:

Ancient Times / Middele Ages / Early Modern / Modern / All

Course program:

Textbooks:

Number of attending students:

Titles of eventual BA or PhD dissertations in progress or discussed:

Student and/or teacher exchange programs activated inside Erasmus/Socrates or other including Urban History courses:

Interested in participating in the Association initiatives and conferences: 


\section{Tables}

Table 1

\begin{tabular}{|c|c|c|c|c|c|c|}
\hline Web search & Denomination & & & & & \\
\hline Faculty & $\begin{array}{c}\text { History of the } \\
\text { City }\end{array}$ & $\begin{array}{l}\text { Landscape and } \\
\text { Settlements }\end{array}$ & Urban History & $\begin{array}{c}\text { History of } \\
\text { Urban Studies }\end{array}$ & Total & $\%$ \\
\hline Architecture & 21 & 1 & 1 & 18 & 41 & 57,7 \\
\hline Humanities & 14 & 6 & & 6 & 26 & 36,6 \\
\hline Economics & & 1 & 1 & & 2 & 2,8 \\
\hline Law & & & 1 & & 1 & 1,4 \\
\hline Engineering & & & & 1 & 1 & 1,4 \\
\hline Total & 35 & 8 & 3 & 25 & 71 & \\
\hline$\%$ & 49,3 & 11,3 & 4,2 & 35,2 & & \\
\hline $\begin{array}{c}\text { Form } \\
\text { collection }\end{array}$ & 11 & 2 & 3 & 6 & 22 & \\
\hline$\%$ & 50 & 9 & 14 & 27 & & \\
\hline
\end{tabular}

Table 2

\begin{tabular}{|c|c|c|c|c|c|}
\hline Web search & Denomination & & & & \\
\hline City & $\begin{array}{c}\text { History of the } \\
\text { City }\end{array}$ & $\begin{array}{c}\text { Landscape and } \\
\text { Settlements }\end{array}$ & Urban History & $\begin{array}{c}\text { History of Urban } \\
\text { Studies }\end{array}$ & Total \\
\hline Rome & 4 & 1 & 1 & 2 & 8 \\
\hline Turin & 3 & 1 & & 4 & 8 \\
\hline Naples & 2 & 1 & & 3 & 6 \\
\hline Venice & 1 & 2 & 1 & 2 & 6 \\
\hline Catania & 4 & & & 1 & 5 \\
\hline Milan & 4 & & & 1 & 5 \\
\hline Bologna & 2 & & & 2 & 4 \\
\hline Ascoli & 1 & & & 2 & 3 \\
\hline Florence & 2 & & & 1 & 3 \\
\hline Genoa & 1 & & 1 & 1 & 3 \\
\hline Ferrara & 2 & & & & 2 \\
\hline Lecce & 1 & & & 1 & . \\
\hline Palermo & 1 & & & 1 & . \\
\hline Perugia & 1 & 1 & & & 7 \\
\hline Pisa & & 1 & & 1 & 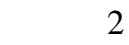 \\
\hline Reggio C. & 1 & & & 1 & 7 \\
\hline Udine & 1 & & & 1 & . \\
\hline Viterbo & 1 & & & 1 & 2 \\
\hline Bari & 1 & & & & 1 \\
\hline Chieti & 1 & & & & 1 \\
\hline Siena & & 1 & & & 1 \\
\hline Trieste & 1 & & & & 1 \\
\hline Tot. & 35 & 8 & 3 & 25 & 71 \\
\hline$\%$ & 49 & 11 & 4 & 35 & \\
\hline
\end{tabular}


Table 3

\begin{tabular}{lrr}
\hline \multicolumn{1}{c}{ City } & Web search & \multicolumn{2}{c}{ Form collection } \\
\hline Rome & 8 & 3 \\
Turin & 8 & 2 \\
Naples & 6 & \\
Venice & 6 & 6 \\
Catania & 5 & 1 \\
Milan & 5 & \\
Bologna & 4 & \\
Ascoli & 3 & \\
Florence & 3 & 2 \\
Genoa & 3 & \\
Ferrara & 2 & \\
Lecce & 2 & \\
Palermo & 2 & 2 \\
Perugia & 2 & 2 \\
Pisa & 2 & \\
Reggio C. & 2 & \\
Udine & 2 & \\
Viterbo & 2 & 1 \\
Bari & 1 & 22 \\
Chieti & 1 & \\
Siena & 1 & \\
Trieste & 1 & \\
\hline Tot. & 71 & \\
\hline
\end{tabular}

Table 4

Form collection

\begin{tabular}{lrrrrrr}
\hline \multicolumn{1}{c}{ Academic position of teachers } & & & \multicolumn{2}{c}{} \\
\hline \multicolumn{1}{c}{ Faculty } & Full Prof. & \multicolumn{1}{c}{ Associate Prof. } & Assistant Prof. & Temporary Prof. & Tot. & \% \\
\hline Architecture & 6 & 1 & 4 & 2 & 13 & 59 \\
Economics & 2 & & & & 2 & 9 \\
Law & & 1 & & 1 & 5 \\
Humanities & & 1 & 4 & 1 & 6 & 27 \\
Tot. & & 3 & 8 & 3 & 22 & \\
$\%$ & 36 & 14 & 36 & 14 & \\
\hline
\end{tabular}

Table 5

\begin{tabular}{lrrr}
\hline $\begin{array}{c}\text { Form } \\
\text { Collection }\end{array}$ & & & \\
\hline \multicolumn{1}{c}{ Level } & N. of courses & & \% \\
\hline Ph.D & 2 & 8 \\
Master & 1 & 4 \\
MA & 6 & 25 \\
BA & 15 & 63 \\
Tot. & 24 & \\
\hline
\end{tabular}


Table 6

Form collection

N. of courses Textbooks

6 G. Zucconi, La città contesa: dagli ingegneri sanitari agli urbanisti (1885-1942), Jaca Book: Milano 1989

3 E. Concina, La città bizantina, Laterza: Bari 2003

3 C. De Seta (ed), Le città capitali, Laterza: Bari 1985

3 H. W. Kruft, Le città utopiche: la città ideale dal XV al XVIII secolo fra utopia e realtà, Laterza: Bari 1990

2 D. Calabi, La città del primo Rinascimento, Laterza: Bari 2001

2 D. Calabi, Storia dell'urbanistica europea, Milano: L. Mondatori 2004

2 D. Calabi, Storia della città: l'età moderna, Marsilio: Venezia 2001

2 Frugoni, Bocchi, Curzi, Pani Ermini, Brogiolo, Guidoni, Iacobini, Cuneo, Città, in Enciclopedia dell'arte medievale, 5, Treccani: Roma 1994

2 L. Gambi, Da città ad area metropolitana, in Storia d'Italia, 5, I documenti, 1, Einaudi: Torino 1973

2 E. Greco, M. Torelli, Storia dell'urbanistica: il mondo greco, Laterza: Bari 1989

2 A. Grohmann, La città medievale, Laterza: Bari 2003

2 P. Gros, M. Torelli, Storia dell'urbanistica: il mondo romano, Laterza: Bari 1992

2 E. Guidoni, A. Marino, Storia dell'urbanistica: il Cinquecento, Laterza: Bari 1982

2 A. Mioni, Le trasformazioni territoriali in Italia nella prima età industriale, Marsilio: Venezia 1986

2 P. Morachiello, La città greca, Laterza: Bari 2003

2 D. Olsen, La città come opera d'arte: Londra, Parigi, Vienna, Milano 1987

2 H. Pirenne, La città del Medioevo, Laterza: Bari 1995

2 B. Zevi, Saper vedere l'urbaniostica: Ferrara di Biagio Rossetti, la prima città moderna europea, Torino 1971

1 M. Agulhon, La ville de l'age industriel, Seuil: Paris 1998

1 Architettura in Europa tra '400 e '800

1 A. Bailly, H. Beguin, Introduzione alla geografia umana, Franco Angeli: Milano 1991

1 P. Battilani, Vacanze di pochi, vacanze di tutti, Il Mulino: Bologna 2001

1 L. Benevolo, Storia della città, Laterza: Bari 1982

1 M. Berengo, La capitale nell'Europa d'antico regime, in C. De Seta (ed), Le città capitali, Laterza: Bari 1985

1 P. Bevilacqua, Tra natura e storia: ambiente, economia e risorse in Italia, Donzelli: Roma 1996

1 P. Bevilacqua (ed), Storia dell'agricoltura italiana in età contemporanea, 1, Spazi e paesaggi, Marsilio: Venezia 1989

1 F. Bocchi, R. Ghizzoni, R. Smurra, Storia delle città italiane dal tardo antico al primo Rinascimento, UTET: Torino 2002

1 L. Bortolotti, Storia, città e territorio, Franco Angeli: Milano 2002

1 R. Bonelli, C. Bozzoni, V. Franchetti Pardo, Storia dell'architettura medievale, Laterza: Bari 2002

1 D. Calabi, Storia della città europea, Venezia: Marsilio 2001

1 R. Camagni, Economia urbana, NIS: Roma 1992

1 A. Fara, La città da guerra nell'Europa moderna, Einaudi: Torino 1993

1 F. Finotto, La città chiusa, Marsilio: Venezia 1992

1 V. Franchetti Pardo, Storia dell'urbanistica: dal Trecento al Quattrocento, Laterza: Bari 1982

1 Gros, La città romana, Laterza: Bari 2004 
1 E. Guidoni, A. Marino, Storia dell'urbanistica: il Seicento, Laterza: Bari 1979

1 P.G. Guzzo, Le città scomparse della Magna Grecia, Newton Compton: Roma 1990

1 P.M. Hohenberg, L'urbanizzazione e le trasformazioni strutturali, in Storia d'Europa, 5, L'età contemporanea (secc. XIX-XX), Einaudi: Torino 1996

1 R. Krautheimer, Tre capitali cristiane: topografia e politica, Einaudi: Torino 1987

1 R. Krautheimer, Architettura paleocristiana e bizantina, Einaudi: Torino 1986

1 F. Lando, L'interpretazione geografica della città, in G. Corna Pellegrini (ed), Aspetti e problemi della geografia, Marzorati: Milano 1987

1 S. Langé, L'eredità romanica, Jaca Book: Milano 1989

1 Le Corbusier, Scritti, a cura di R. Tamburrino, Einaudi: Torino 2003

1 M. Manieri Elia, Architettura e mentalità (dal Classico al Neoclassico), Laterza: Bari 1989

1 F. Martinelli, La città: i classici della sociologia, Liguori: Napoli 2002

1 C. Norberg-Schulz, Architettura barocca, Electa: Milano 1971

1 A. Restucci, Città e architetture nell'Ottocento, in Storia dell'arte italiana, II, 2, Einaudi: Torino 1982

1 M. Roncayolo, La città, Einaudi: Torino 1988

1 P. Sica, Storia dell'urbanistica: l'Ottocento, 1, Laterza: Bari 1991

1 G. Simoncini, La città nell'età dell'Illuminismo: le capitali italiane, Firenze: Olschki 1996

1 C. Sitte, L'arte di costruire le città, Jaca Book, Milano 1980

1 Storia dell'architettura e delle tecniche costruttive dal XV al XVIII secolo

1 F. Dal Co (ed), Storia dell'architettura italiana, Electa: Milano 2003-2004

1 M. Tafuri, Venezia e il Rinascimento, Einaudi: Torino 1985

1 M. Tafuri, F. Dal Co, Architettura contemporanea, Electa: Milano 1976

1 Urbanistica nel Mezzogiorno moderno e contemporaneo

1 P. Villani, La città europea nell'età industriale, in P. Rossi (ed), Modelli di città, Einaudi: Torino 1987

1 G. Zucconi, La città dell'Ottocento, Laterza: Bari 2000

\begin{tabular}{cccc} 
Textbooks & N. of courses & Mean & \\
\hline 83 & & 24 & 3,5 \\
\hline
\end{tabular}

